\title{
Valor da Biópsia de Pericárdio no Diagnóstico Etiológico das Pericardiopatias
}

\author{
Fábio Fernandes, Barbara Maria Ianni, Edmundo Arteaga, Luis Benvenutti, Charles Mady
}

São Paulo, SP

Objetivo - Determinar o valor da biópsia no diagnóstico etiológico de derrames pericárdicos.

Métodos - Foram analisados, retrospectivamente, de 1990 a 1997, 38 pacientes com derrame pericárdico, com

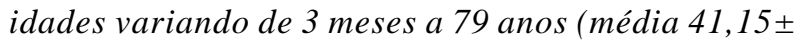
21,78 anos). Todos os pacientes foram submetidos a biópsia pericárdica, tendo como acesso a via subxifóide.

Resultados - A biópsia foi positiva em quatro $(10,5 \%)$ casos, dois de etiologia tuberculosa e dois neoplásica (um adenocarcinoma indeterminado e um mesotelioma); no restante o diagnóstico foi de pericardite crônica inespecífica.

Conclusão - Em nossa casuística a biópsia não foi um método eficaz para o diagnóstico etiológico dos derrames pericárdicos. Acreditamos que a indicação de biópsia pericárdica deva ser individualizada, especificamente nos casos de suspeita de etiologia neoplásica ou tuberculosa.

Palavras-chave: pericardite, biópsia, etiologia

\section{Value of Pericardial Biopsy in the Etiologic Diagnosis of Pericarditis}

Purpose - To determine the value of pericardial biopsy as a complementary exam in the etiology of pericardial effusion.

Methods - We analyzed retrospectively (from 1990 to 1997) 38 patients with pericardial effusion. The age ranged between 3 months and 79 years (mean $41.15 \pm$ 21.78 years). Pericardial biopsy was perfomed in all cases through a subxiphoid incision.

Results - In 4 patients (10.5\%) the biopsy was able to define etiology, 2 had tuberculosis and 2 neoplasias ( 1 undetermined adenocarcinoma, 1 mesotelioma). In 34 patients the biopsy showed inespecific chronic pericarditis.

Conclusion - Pericardial biopsy was not an effective method to determine the etiological diagnosis in pericardial effusion patients. We believe that we have to individualise the indication of pericardial biopsy especially if tuberculosis or neoplasia are suspected.

Key-words: pericarditis, biopsy, etiology

Arq Bras Cardiol, volume 70 (nº 6), 393-395, 1998

As doenças do pericárdio têm várias etiologias, levando a diferentes tipos de acometimento morfológico. Apesar do quadro clínico e, atualmente, dos exames não invasivos permitirem diagnóstico acurado de derrames e constrições pericárdicas, não temos a mesma facilidade, em se tratando do diagnóstico etiológico ${ }^{1}$. Métodos complementares invasivos têm sido utilizados para a detecção das causas das pericardites, principalmente a biópsia pericárdica. Este procedimento é considerado técnica de rotina, em vários centros, embora sua indicação varie de um para outro, não

Instituto do Coração do Hospital das Clínicas - FMUSP

Correspondência: Fabio Fernandes - Incor - Divisão Clínica - Av. Dr. Enéas C. Aguiar, 44 - 05403-000 - São Paulo, SP

Recebido para publicação em 9/1/98

Aceito em 19/3/98 existindo uniformidade quanto ao valor do método. Os resultados discordantes, provavelmente, são consequência não só da variação das indicações, mas também das casuísticas diferentes, relatadas na literatura. Portanto, é importante que cada grupo tenha sua própria experiência.

O objetivo desse trabalho foi determinar, em nosso meio, o valor da biópsia pericárdica, como método de diagnóstico etiológico de derrames pericárdicos.

\section{Métodos}

No Incor, de 1992 a 1997, foram analisados, retrospectivamente, 38 pacientes com derrame pericárdico, submetidos a biópsia na tentativa de detecção da etiologia do processo. Eram 20 do sexo masculino, com idades entre 3 meses e 79 anos (média 41,15 $\pm 21,78$ anos). Após avaliação clínica, 
o tipo de comprometimento foi caracterizado pelo ecocardiograma e, se necessário, complementado pela tomografia de tórax e ressonância nuclear magnética. A via de acesso utilizada para a biópsia pericárdica foi a subxifóide, através de incisão longitudinal mediana de $3 \mathrm{~cm}$, iniciando cranialmente na extremidade distal do corpo do esterno. Um fragmento de aproximadamente $1 \mathrm{~cm}^{2}$ do pericárdio foi ressecado e enviado para exame anatomopatológico. O material obtido foi fixado e examinado à microscopia óptica, depois de corado pelos métodos da hematoxilina-eosina, ZiehlNielsen, Grocotte BH.

\section{Resultados}

Dos 38 pacientes, quatro $(10,5 \%)$ tiveram a etiologia definida pela biópsia, sendo dois por tuberculose (fig. 1) e dois por neoplasias (um adenocarcinoma indeterminado e um mesotelioma), ficando o restante $(89,5 \%)$ com diagnóstico anatomopatológico de pericardite crônica inespecífica (fig. 2).

\section{Discussão}

A doença pericárdica tem causas variadas ${ }^{2}$. A diferença significativa de diagnósticos etiológicos parece ter correlação com a região geográfica e diferentes populações ${ }^{3}$.

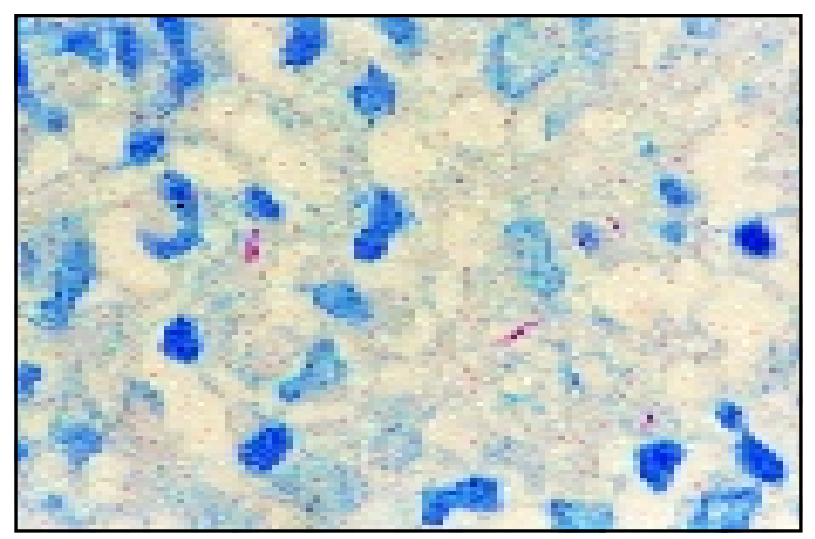

Fig. 1 - Bacilos álcool-ácido resistentes (BAAR) presentes em um caso de pericardite tuberculosa (Ziehl-Nielsen, x400).

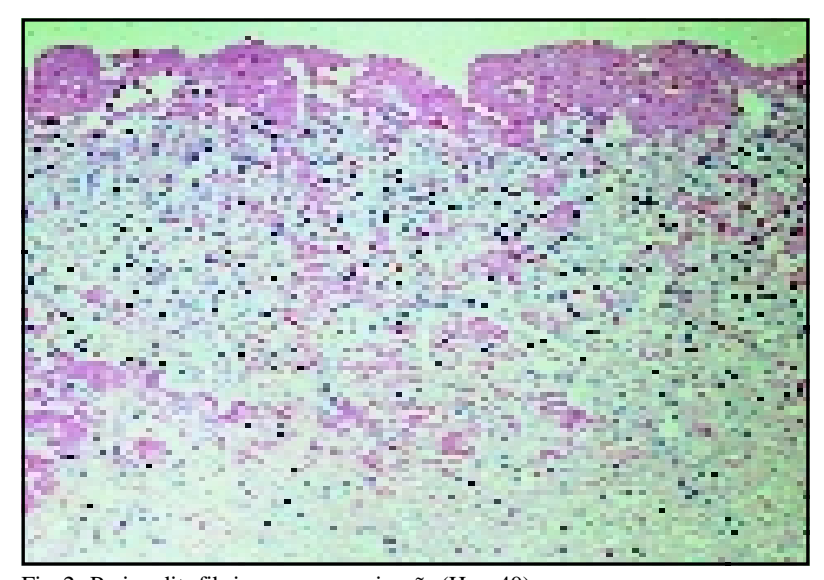

Fig. 2 - Pericardite fibrinosa em organização $(\mathrm{He}, \mathrm{x} 40)$.
Apesar da propedêutica clínica e dos métodos complementares atuais terem aumentado a freqüência de diagnóstico das pericardiopatias, o mesmo não ocorreu em relação à determinação da causa do comprometimento.

A decisão de se adotar uma técnica invasiva na pesquisa da etiologia da pericardite é basicamente influenciada pelo quadro clínico do paciente. Quando este é severo, com queda da pressão sangüínea e dispnéia, a drenagem pericárdica é indicada, a despeito da etiologia. Por outro lado, quando a etiologia é incerta, a biópsia pode ser indicada para fins diagnósticos. A identificação da etiologia da doença pericárdica pelo derrame é difícil, uma vez que existem limitações citológicas e microbiológicas na análise do líquido pericárdico. Em relação à biópsia do pericárdio, a grande limitação deve-se à dificuldade em se obter tecido pericárdico adequado para o diagnóstico anatomopatológico ${ }^{4}$.

Quanto ao valor da biópsia no diagnóstico etiológico das pericardiopatias, a literatura disponível é apenas parcialmente esclarecedora.

Zayas e col ${ }^{5}$, estudando os métodos complementares para diagnóstico etiológico em 100 pacientes com pericardite aguda, definiram a etiologia em $22(22 \%)$ pacientes. A biópsia pericárdica foi negativa nos cinco casos em que foi realizada, não contribuindo para o diagnóstico etiológico.

Com a finalidade de avaliar a eficácia clínica da pericardiocentese e da biópsia pericárdica, Sagristà-Sauleda e $\mathrm{col}^{6}$ realizaram biópsia com finalidade diagnóstica em 25 pacientes e concomitante à punção nos casos sintomáticos (finalidade terapêutica) em 11. A biópsia diagnóstica foi positiva em apenas um caso, sendo feito o diagnóstico de tuberculose. Nos casos da biópsia terapêutica a positividade foi maior (seis casos): em três, neoplasias; em dois, infecção e em um, tuberculose. O diagnóstico etiológico foi feito pela biópsia em $19 \%$ ( 7 de 36) com sensibilidade de $77 \%$ e especificidade de $96 \%$. Esses autores concluíram que a pericardiocentese e a biópsia terapêutica devem ser individualizadas e sugerem que em uma comunidade de elevada prevalência de tuberculose a biópsia deve ser procedimento de eleição.

Corey e $\operatorname{col}^{1}{ }^{1}$, em estudo que avaliou a eficácia da biópsia no diagnóstico etiológico de pacientes com derrame pericárdico, encontraram etiologia definida em $93 \%$ dos pacientes, sendo em $26 \%$ dos casos pela punção e em $23 \%$ pela biópsia. Concluíram que tanto a punção como a biópsia são elementos que auxiliam no diagnóstico das pericardites.

Em nosso meio, Vianna e $\mathrm{col}^{7}$, estudando oito casos de pericardite tuberculosa, encontraram estudo histopatológico compatível com tuberculose em quatro, concluindo ser a biópsia pericárdica uma importante contribuição diagnóstica. Por sua vez, Ianni e col ${ }^{8}$, estudando a biópsia no diagnóstico do comprometimento pericárdico por tumores malignos, mostraram que em $41 \%$ dos casos o diagnóstico foi compatível com o comprometimento e concluíram que a biópsia é método seguro e de alta especificidade, porém de baixa sensibilidade. 
Com relação aos dois últimos trabalhos citados, duas questões merecem ser discutidas: $1^{a}$ ) foram selecionados grupos específicos de pacientes nos quais o diagnóstico etiológico provável era elevado, ou seja, a biópsia apresentava alto valor preditivo positivo; $2^{\mathrm{a}}$ os resultados positivos da biópsia se devem, basicamente, à etiologia tuberculosa ou neoplásica, que leva, alguns centros a preconizar a biópsia de rotina em grandes derrames de causa indeterminada, particularmente, se houver suspeita de etiologia tuberculosa ou neoplásica ${ }^{7}$.

Em nosso estudo, a biópsia foi positiva apenas em $10,5 \%$ dos casos. No restante, o diagnóstico foi de pericardite crônica inespecífica, talvez refletindo um grupo não selecionado de pacientes. Porém, qual deveria ser nossa conduta com relação a esses doentes? Se, por um lado, a possibilidade diagnóstica foi pequena, por outro, ajudou na abordagem desse grupo de pacientes que, afinal, não foi desprezível. Talvez, por uma avaliação clínica complementar não invasiva, pudéssemos selecionar subgrupos de pacientes em que a biópsia traria informações importantes para a terapêutica, principalmente, em relação à tuberculose e neoplasias.

Com a finalidade de aumentar a sensibilidade da biópsia surgiram novas estratégias diagnósticas e terapêuticas, como por exemplo, a videotoracoscopia. Esta técnica foi possível pelo desenvolvimento de novos equipamentos vídeo-endoscópicos, aliados à instrumentação cirúrgica endoscópica, que expandiu a toracoscopia de uma modalidade diagnóstica para um procedimento minimamente invasivo $^{9}$. Assim, sob visão direta, observam-se as regiões pericárdicas mais acometidas, acesso direto antes permitido, somente, a patologistas e cirurgiões. Com tal método, pode-se estabelecer um novo marco na classificação macroscópica das doenças pericárdicas, acrescido de informações microscópicas ${ }^{10}$. É considerada também uma técnica segura ${ }^{11} \mathrm{e}$, freqüentemente, acompanhada da necessidade de pericardiocentese para drenagem. Acreditamos que a pericardioscopia, com o tempo, possa contribuir para aumentar a positividade do diagnóstico etiológico das pericardiopatias.

Em conclusão, na nossa casuística, a biópsia de pericárdio por via subxifóide revelou a etiologia do processo em $10,5 \%$, porcentagem pequena, porém não desprezível, tendo em conta a possibilidade do tratamento dirigido à causa. Dessa forma, antes de indicar a biópsia pericárdica, devemos esgotar toda avaliação diagnóstica complementar, lembrando que outros métodos não permitem o diagnóstico etiológico, mas apenas de probabilidade. Devemos também individualizar as indicações, especialmente se as etiologias neoplásica ou tuberculosa forem suspeitadas.

\section{Referências}

1. Corey GR, Campbell PT, Trigt PV et al -Etiology of large pericardial effusion. Am J Cardiol 1993; 95: 209-13.

2. Maisch B, Drude L - Pericardioscopy- a new diagnóstic tool in inflammatory diseases of the pericardium. Eur Heart J 1991; 12 (supplement D): 2-6.

3. Ilan Y, Oren R, Chetrit EB - Etiology, treatment and prognosis of large pericardial effusions. Chest 1991; 100: 985-7.

4. Ziskind AA, Rodriguez S, Lemmon C, Burstein S-Percutaneous pericardial biopsy as an adjunctive technique for the diagnosis of pericardial disease.Am J Cardiol 1994; 74: 288-91.

5. Zayas R, Aguita M, Torres Fet al - Incidence of especific etiology and role of methods for especific etiologic diagnosis of primary acute pericarditis. Am J Cardiol 1995, 75: 378-82.

6. Sagistá-Sauleda J, Miralda GP, Sler JSS - Eficácia clínica da pericardiocentese e biópsia pericárdica. Rev Esp Cardiol 1987, 40: 94-9.

7. Vianna CB, Barreto ACP, Mady C et al - Pericardite tuberculosa. Dificuldade diagnósticas e valor da biópsia pericárdica. Arq Bras Cardiol 1986; 47: 27-30.

8. Ianni BM, Barreto ACP, Mady C et al - A biópsia no diagnóstico do comprometimento pericárdico por tumores malignos. Arq Bras Cardiol 1989; 53: 157-9.

9. Mack MJ,Landreneau RJ, Hazelrigg SR, Acuff TE - Video thoracoscopic management of benign and malignant pericardial effusions. Chest 1993; 103: 390s-3s.

10. Maisch B, Bethege C, Drude L, Hufnagel G, Herzum M, Schonian U - Pericardioscopy and epicardial biopsy- new diagnostic tools in pericardial and perimyocardial disease. Eur Heart J 1994; 15(supplement C): 68-73.

11. Millaire A, Wurtz A, Groote P, Saudemont A, Chambon A, Ducloux G - Malignant pericardial effusions: Usefulness of pericardioscopy. Am Heart J 1992, 124 : 1030-4. 